\title{
Crystal structure of $\beta$-arrestin 2 in complex with an atypical chemokine receptor phosphopeptide reveals an alternative active conformation
}

Kyungjin Min ${ }^{\mathrm{a}, 1}$, Hye-Jin Yoon ${ }^{\mathrm{a}, 1}$, Ji Young Park ${ }^{\mathrm{b}}$, Mithu Baidya ${ }^{\mathrm{c}}$, Hemlata Dwivedi ${ }^{\mathrm{c}}$, Jagannath Maharana $^{\mathrm{c}}$, Ka Young Chung ${ }^{\mathrm{b}, 2}$, Arun K. Shukla ${ }^{\mathrm{c}, 2}$, Hyung Ho Lee $\mathrm{e}^{\mathrm{a}, 2}$

${ }^{a}$ Department of Chemistry, College of Natural Sciences, Seoul National University, Seoul 08826, Republic of Korea

${ }^{\mathrm{b} S c h o o l ~ o f ~ P h a r m a c y, ~ S u n g k y u n k w a n ~ U n i v e r s i t y, ~ S u w o n, ~ 16419, ~ R e p u b l i c ~ o f ~ K o r e a . ~}$

${ }^{\mathrm{c} B i o l o g i c a l ~ S c i e n c e s ~ a n d ~ B i o e n g i n e e r i n g, ~ I n d i a n ~ I n s t i t u t e ~ o f ~ T e c h n o l o g y, ~ K a n p u r, ~ I n d i a ~}$

${ }^{1}$ These authors contributed equally

2Correspondence: kychung2@skku.edu; arshukla@iitk.ac.in; hyungholee@snu.ac.kr

Key words: Cellular signaling, G protein-coupled receptors (GPCRs), atypical chemokine receptors (ACKRs), $\beta$-arrestins, CXCR7, biased agonism, synthetic antibody fragments 


\section{Abstract}

$\beta$-arrestins ( $\beta$ arrs) critically regulate signaling and trafficking of $\mathrm{G}$ protein-coupled receptors (GPCRs), the largest family of drug targets in the human genome, and there are two isoforms of ßarrs: $\beta$ arr1 and $\beta$ arr2. Most GPCRs interact with both the heterotrimeric G-proteins and $\beta$ arrs, inducing distinct downstream signal transduction. However, certain chemokine receptors lack functional G-protein coupling, but they can efficiently recruit ßarrs upon agonist-stimulation, and they are referred to as atypical chemokine receptors (ACKRs). Receptor phosphorylation is a key determinant for the binding of $\beta$ arrs, and understanding the intricate details of receptor- $\beta$ arr interaction is the next frontier in GPCR structural biology. To date, the high-resolution structures of active $\beta$ arr1 have been revealed, but the activation mechanism of $\beta$ arr2 by a phosphorylated GPCR remains elusive. Here, we present a $1.95 \AA$ crystal structure of $\beta$ arr2 in complex with a phosphopeptide (C7pp) derived from the carboxyl-terminus of ACKR3, also known as CXCR7. The structure of C7pp-bound $\beta$ arr2 reveals key differences from the previously determined active conformation of $\beta$ arr1. One of the key differences is that C7pp-bound $\beta$ arr2 shows a relatively small inter-domain rotation. An antibody-fragment-based conformational sensor and hydrogen/deuterium exchange experiments further corroborate structural features and suggest that the determined structure is an alternative active conformation of $\beta$ arr2. 


\section{Introduction}

G-protein-coupled receptors (GPCRs) are the largest family of receptors on cell membranes and comprise an important class of drug targets. In response to ligand binding, GPCRs activate G proteins as a guanine nucleotide exchange factor, which triggers downstream signaling. To turn off G-protein-mediated GPCR signaling, GPCR kinases phosphorylate the C-terminal tail and/or intracellular loops of GPCRs, which leads to arrestin binding. Although there are over 800 GPCRs in the human genome, only four arrestin genes (arrestins 1-4) have been identified. Among the four arrestin subtypes, arrestin-1 and arrestin- 4 are solely related to rhodopsin and cone opsin in the visual system, while arrestin-2 and arrestin-3 ( $\beta$-arrestin-1 and $\beta$-arrestin-2, hereafter $\beta$ arr1 and $\beta$ arr2, respectively) are ubiquitously expressed, and they are responsible for interaction with and regulation of nonvisual GPCRs. The interaction of $\beta$ arrs with phosphorylated receptors transits $\beta$ arrs to their active state, which leads to desensitization and/or internalization of GPCRs. It is also well established that $\beta$ arrs critically contribute in a range of downstream signaling responses for many different GPCRs ${ }^{1}$. In addition, $\beta$ arrs are also recognized as multifunctional and versatile adaptor proteins that bind to and regulate dozens of non-receptor proteins as well ${ }^{2}$.

The interaction of GPCRs and $\beta$ arrs is typically a two-step process that involves docking of the phosphorylated receptor tail (i.e., the carboxyl-terminus) to the N-domain of $\beta$ arrs and the interaction of the receptor core (i.e., the intracellular side of the receptor transmembrane bundle) with loops on the convex side of $\beta$ arrs. While the primary cellular functions of $\beta$ arrs are broadly conserved across different GPCRs, there is increasing evidence for receptor-specific fine-tuning of $\beta$ arr functions. Although a clear mechanism for functional diversity of $\beta$ arrs remains mostly 
elusive, it has been proposed that different patterns of receptor phosphorylation establish distinct phospho-barcodes on the receptor that fine-tune the interaction pattern and conformational signatures of $\beta$ arrs, resulting in specific functions ${ }^{3-6}$. To decode how distinct phosphorylation patterns govern $\beta$ arr conformations and functional outputs, it is essential to visualize the structural details of $\beta$ arrs in complex with differentially phosphorylated GPCRs or their corresponding phosphopeptides.

There has been significant effort in the recent years to understand the molecular mechanism of $\beta$ arr activation, including crystal structures of pre-activated arrestin- $1^{7}$, $\beta$ arr 1 in complex with the phosphorylated vasopressin receptor tail $\left(\mathrm{V}_{2} \mathrm{Rpp}\right)^{8}$, and a rhodopsin-arrestin-1 fusion protein $^{9,10}$. These structures have revealed major conformational changes upon arrestin-1 and $\beta$ arrl activation, such as a significant inter-domain rotation $\left(\sim 20^{\circ}\right)$, disruption of threeelement (3E) and polar-core interactions, and reorientation of various loops, including the finger and lariat loops. The $\mathrm{V}_{2} \mathrm{Rpp}$-bound $\beta$ arrl structure also confirmed a previously suggested molecular mechanism, in which the binding of the phosphorylated receptor tail to the N-domain of an arrestin displaces its carboxyl-terminus (Fig. 1a) ${ }^{11,12}$. Furthermore, the crystal structure of a rhodopsin-arrestin-1 fusion protein has also provided structural details for a fully engaged complex, including the interface between arrestin-1 and the receptor core, in addition to a phosphorylation-dependent interaction ${ }^{9,10}$. Single-particle negative-staining-based electron microscopy has also provided direct visualization of the biphasic interaction between the receptor and $\beta$ arr1 by capturing partially engaged (associated through the receptor tail) and fully engaged (involving the receptor core) complexes ${ }^{13}$.

However, activation of $\beta$ arr2 by the phosphorylated receptor and how it differs from that of $\beta$ arr1 remain to be structurally visualized. This is particularly important considering that 
despite ubiquitous expression and high sequence similarity, $\beta$ arr1 and $\beta$ arr2 display a significant level of functional divergence ${ }^{1,14}$. For example, some GPCRs bind $\beta$ arr2 with higher affinity than Barr1 while others recruit both isoforms with similar affinities ${ }^{15}$. Moreover, in some cases, the two isoforms of ßarrs also contribute differently toward their conserved functions of receptor desensitization, endocytosis, and signaling ${ }^{14}$. Additionally, for some receptors such as the bradykinin and angiotensin receptors, depletion of $\beta$ arr2 results in a decrease of agonist-induced ERK1/2 MAP kinase phosphorylation, while depletion of $\beta$ arr1 enhances it ${ }^{16,17}$. Thus, to fully understand $\beta$ arr-mediated GPCR regulation and to delineate their functional divergence, visualization of the structural details of $\beta$ arr2 activation is essential.

Accordingly, in this study we focus on capturing active conformations of $\beta$ arr2 in complex with phosphopeptides originating from the carboxyl-terminus of a chemokine receptor, CXCR7, also referred to as atypical chemokine receptor 3 (ACKR3). CXCR7, a Class A GPCR, forms a heterodimer with another chemokine receptor, CXCR4, and it has been proposed that it acts as a "scavenger" of its chemokine ligand, CXCL12 18 . It has also been suggested that CXCR7 may represent a natural example of a $\beta$ arr-biased 7TM receptor because it interacts with ßarrs but does not display functional-coupling with heterotrimeric G-proteins ${ }^{18}$. Here, we determine the crystal structure of $\beta$ arr2 in complex with a CXCR7 phosphopeptide, and the structure reveals key differences with the previously determined structure of $\mathrm{V}_{2} \mathrm{Rpp}$-bound $\beta$ arr 1 . In addition, we also utilize a diverse set of complementary biochemical and biophysical approaches, including site-directed mutagenesis, hydrogen/deuterium exchange mass spectrometry (HDX-MS), and synthetic-antibody-based conformational sensors to extract insights into the activation of $\beta$ arr2. 


\section{Results and discussion}

Agonist-induced $\beta$-arrestin recruitment and trafficking for CXCR7. As mentioned earlier, CXCR7 does not exhibit functional coupling to any of the major sub-types of heterotrimeric Gproteins, although it does efficiently couple to $\beta$ arr2. We first validated $\beta$ arr2 coupling and trafficking in HEK-293 cells using a PRESTO-TANGO assay ${ }^{19}$ and confocal microscopy (Fig. 1b-c). We observed that CXCR7 efficiently recruits $\beta$ arr2 and behaves as a Class B receptor in terms of its trafficking pattern of $\beta$ arr2 (i.e., receptors are internalized). Based on a recent study that proposed the importance of different phosphorylation codes in GPCRs for $\beta$ arr binding ${ }^{10}$, we identified two potential phosphorylation-codes in the carboxyl-terminus of CXCR7 with PxxPxxP (pSAKpTGLpT) and PxPxxP (pSEpTEYpS) patterns (Fig. 1d). To identify which phosphorylation code is responsible for $\beta$ arr2 recruitment to CXCR7, we generated two different CXCR7 phosphorylation-code mutants, referred to as $\mathrm{CXCR} 7^{\text {mut } 1}$ and $\mathrm{CXCR} 7^{\text {mut2 }}$, that eliminated these two codes sequentially (Fig. 1d). Subsequently, we measured the interaction and trafficking of $\beta$ arr2 with these two receptor mutants (Fig. 1e-f). We observed that CXCR7 ${ }^{\text {mut1 }}$ behaved essentially similar to a wild-type receptor, while CXCR7 $7^{\text {mut2 }}$ exhibited a substantial reduction in $\beta$ arr2 recruitment and trafficking (Fig. 1f). These findings underscore the relatively important contribution of the second cluster, i.e., the PxPxxP motif, in the $\beta$ arr2 interaction with CXCR7. A previous study has also demonstrated that the second cluster is more responsible for the internalization and degradation of CXCL12 through CXCR7 than the first cluster ${ }^{20}$.

Generation and characterization of CXCR7 phosphopeptides. Although the second phosphorylation-code (PxPxxP) was critical for $\beta$ arr2 recruitment, we synthesized two different 
phosphopeptides, referred to as C7pp1 and C7pp2, to investigate their interaction with $\beta$ arr2 and any corresponding structural changes (Fig. 2a). These peptides harbor PxxPxxP and PxPxxP patterns of phosphorylation, respectively (Fig. 2a), and may give us insight into the phosphocode-dependent structural changes of $\beta$ arr2. These two phosphopeptides exhibited similar binding affinities to $\beta$ arr2, with dissociation constants $\left(K_{\mathrm{D}}\right)$ of $3.08 \pm 0.3 \mu \mathrm{M}$ for $\mathrm{C} 7 \mathrm{pp} 1$ and $0.581 \pm 0.03 \mu \mathrm{M}$ for $\mathrm{C} 7 \mathrm{pp} 2$, as measured by isothermal titration calorimetry (Fig. 2b). Interestingly however, while C7pp1 displays a monophasic binding with $\beta$ arr2, the binding parameters of C7pp2 display a better fit using a two-site model, and future studies are necessary to understand the biological significance of this observation, if any.

To understand the structural changes of $\beta$ arr2 upon $\mathrm{C} 7 \mathrm{pp} 1$ or $\mathrm{C} 7 \mathrm{pp} 2$ binding, we performed HDX-MS (Fig. 2c). HDX-MS monitored the exchange between the amide hydrogen of a protein and deuterium in the solvent, and the exchange rate was dependent on the solvent exposure and conformational flexibility of the amide hydrogen. The HDX-MS profiles of $\beta$ arr2 with or without co-incubation of C7pp1 or C7pp2 were analyzed, which showed that C7pp1 and C7pp2 binding induced iconic changes of active arrestins. We observed increased HDX within residues 383-390 containing $\beta$ XX and residues 292-301 containing the gate loop (the C-terminal part of the lariat loop), implying release of the C-terminus and disruption of the polar core. Additionally, we observed decreased HDX within residues 119-133 containing the middle loop, residues 283-291 containing the $\mathrm{N}$-terminal part of the lariat loop, and residues 305-317 containing the back loop, which may imply the movement of the inter-domain regions. Interestingly, C7pp1 and C7pp2 induced similar HDX changes, suggesting that the overall conformational dynamics between C7pp1- and C7pp2-bound ßarr2 were similar although the detailed atomic structures may have differed. 
Crystal structure of phosphopeptide-bound Barr2. To reveal the atomic details of C7pp1- or C7pp2-bound ßarr2, we performed X-ray crystallography to obtain high-resolution structures. Although C7pp1 and C7pp2 bind efficiently to the full-length $\beta$ arr2 (Fig. 2b-c), we used a truncated version of $\beta$ arr2 lacking the carboxyl-terminal residues 357-410 to facilitate the crystallization of $\beta$ arr2 in an active conformation. Unfortunately, the crystals of C7pp2-bound ßarr2 did not diffract well, whereas we successfully obtained at $1.95 \AA$ resolution the crystal structure of $\beta$ arr2 in complex with C7pp1 (Fig. 2d and Supplementary Fig. 1a). Therefore, we focus our discussion on the conformational details of C7pp1-bound $\beta$ arr2.

The crystals of C7pp1-bound $\beta$ arr2 appeared to be pseudo-merohedrally twinned in the $C 2_{1}$ space group with a high $\mathrm{R}_{\text {merge }}$ value, and thus the structure was refined with detwinned data (Supplementary Table 1). The electron density map of residues 331-332 was not observed for C7pp1 (chain U), while nearly all sequences of $\beta$ arr2 were found to be ordered with the exception of the internal flexible regions (residues 175-181 in chain $\mathrm{C}$ and $\mathrm{F}$, respectively) (Supplementary Fig. 1). C7pp1 adopts an elongated loop over the entire length $(\sim 35 \AA)$ without severe kinking and is paired with the highly cationic concave surface of the $\beta$ arr2 $\mathrm{N}$-domain, with a total surface area of $928.4 \AA^{2}$ buried at the interface (Supplementary Fig. 2).

While interpreting the structural changes in $\beta$ arr2 upon C7pp1 binding, especially in terms of comparing them with inactive $\beta$ arr2 or that of $\mathrm{V}_{2} \mathrm{Rpp}$-bound $\beta$ arr1, caution is warranted when analyzing the regions involved in crystal contacts that may lead to crystallographic artifacts. Interestingly, the crystallographic asymmetric unit of the ßarr2-C7pp1 complex contains six heterodimers of $\beta$ arr2 and C7pp1, and it shows that the crystallographic contacts of the six molecules are not identical to each other (Supplementary Fig. 1a). Thus, we are able to find at least one solvent-exposed region amongst the six molecules for activation-dependent 
regions, which allows us to confidently interpret the C7pp1-induced structural changes in $\beta$ arr2. Moreover, the six $\beta$ arr2-C7pp1 molecules show essentially similar structures overall when they are superimposed (average root-mean-square deviation of $1.138 \AA$ for the $334 \mathrm{C} \alpha$ atom pairs) (Supplementary Fig. 1b).

\section{Smaller inter-domain rotation of $\mathbf{C 7 p p 1 - \beta a r r 2}$ compared to $\mathbf{V}_{2} \mathbf{R p p - \beta a r r 1 . ~ T h e ~ C 7 p p 1 - ~}$} bound $\beta$ arr2 structure shares conformational changes similar to other existing active arrestin structures, such as the disruption of the $3 \mathrm{E}$ interaction and the polar-core interaction (discussed in Fig. 4b-c). However, the most striking differences between C7pp1-bound ßarr2 and other active structures of arrestin-1 or $\beta$ arrl is found in the inter-domain rotation angle (Fig. 3a). Preactivation of arrestin- 1 or $\mathrm{V}_{2} \mathrm{Rpp}$ binding to $\beta$ arr1 induce $\sim 21^{\circ}$ and $\sim 20^{\circ}$ inter-domain rotation, respectively ${ }^{7,8}$. Even $\mathrm{IP}_{6}$-bound $\beta$ arr2 exhibits $\sim 17^{\circ}$ of inter-domain rotation ${ }^{21}$. Like these structural changes, compared to the inactive $\beta$ arr2 state $\beta$ arr2 underwent inter-domain rotation in the C7pp1-bound structure (Fig. 3a). However, the inter-domain rotation angle of C7pp1-bound $\beta$ arr2 is significantly smaller $\left(\sim 8^{\circ}\right)$ than that of $\beta$ arr $1\left(\sim 20^{\circ}\right)$ when they are in their final states (Fig. 3a). This data leads us two hypotheses: first, unlike arrestin-1 or $\beta$ arr1, the receptor-bound Barr2 adopts a structure with smaller inter-domain rotation when it interacts with a phosphorylated receptor C-tail; and second, $\beta$ arr2 adopts structures with various inter-domain rotations depending on the binding partners.

To test the first hypothesis, we measured the reactivity of a conformationally selective antibody fragment, Fab30, toward C7pp1-, C7pp2-, and $\mathrm{V}_{2}$ Rpp-bound $\beta$ arr2. Fab30 efficiently interacted with $\mathrm{V}_{2} \mathrm{Rpp}$-bound $\beta$ arr1 and $\beta$ arr 2, and molecular dynamics simulations have suggested that an inter-domain rotation of more than $15^{\circ}$ is most optimal for Fab30 reactivity ${ }^{22}$. 
We did not observe a significant interaction of Fab30 with C7pp1-bound $\beta$ arr2 (Fig. 3b-c). This was consistent with the smaller inter-domain rotation observed in the C7pp1-bound crystal structure of $\beta$ arr2 (Fig. 3a). However, Fab30 interacted robustly with $\mathrm{V}_{2}$ Rpp-bound $\beta$ arr2 (Fig. 3b-c). Interestingly, Fab30 also did not interact with C7pp2-bound ßarr2 (Fig. 3b-c), which was consistent with the HDX-MS data showing similar conformational dynamics near the interdomain region between C7pp1- and C7pp2-bound ßarr2 (Fig. 2c, residues 119-133, 283-291, 292-301, 305-317, and 383-390). These results suggested that $\beta$ arr2 adopts different conformations when bound to different $\mathrm{R}_{\mathrm{p}}$-tails or different activation stimuli, thus rejecting the first hypothesis - the smaller inter-domain rotation in C7pp1-bound $\beta$ arr2 structure may indicate an inherent propensity specific to $\beta$ arr2 upon activation.

An alternative hypothesis is that specific phosphorylation patterns, i.e., the number and spatial distribution of phosphates, govern the inter-domain rotation and thereby impart corresponding functional conformation on $\beta$ arr2. Although such a possibility remains to be explored further, it may explain not only the structural basis of the bar-code hypothesis but also receptor-specific functional outcomes of $\beta$ arrs. Therefore, we suggest that the current C7pp1bound $\beta$ arr2 structure represents an alternative active conformation that may be observed for other receptors as well, depending on the specific phosphorylation pattern. Considering that even partially engaged receptor-ßarr conformations are functionally competent, for example, in terms of mediating receptor endocytosis and ERK1/2 MAP kinase activation ${ }^{21,23}$, the current structure has direct implications for understanding the structural details of receptor- $\beta$ arr interaction, and for ensuring functional responses. It should also be noted that the structure represents the $\beta$ arr2 conformation in complex with an isolated phosphopeptide without including the core interaction 
with the receptor. It is also plausible that the core interaction may further fine-tune the conformation of $\beta$ arr2 including the inter-domain rotation angle.

Distinct conformational changes of the loop regions in the C7pp1-ßarr2 structure. To gain further structural insights into the conformation of $\beta$ arr2 induced by $\mathrm{C} 7 \mathrm{pp} 1$ binding, the active Barr1 structure in complex with $\mathrm{V}_{2} \mathrm{Rpp}$ (4JQI) was compared with the structure of our $\beta$ arr2C7pp1 complex (Fig. 4). As discussed above, the N-domain and central loops showed large conformational changes upon activation ${ }^{24}$. The loop regions underwent conformational changes upon C7pp1 binding, and the structures were different in several ways from those of $\mathrm{V}_{2} \mathrm{Rpp}$ bound Barr1 (Fig. 4a). First, the C7pp1 peptide occluded the inactive conformation of the finger loop lock, promoted outward movement, and induced a helical structure in our crystal structure (Fig. 4a, left panel). This was surprising because the finger loop of the $\beta$ arr $1-\mathrm{V}_{2}$ Rpp complex exhibited an extended conformation ${ }^{8}$, and the helical structure of the finger loop was observed when the arrestin was fully docked to the GPCR core. However, it should be noted that HDXMS analysis did not indicate a helix formation in the finger loop (Fig. 2c), suggesting that the helix observed in the current structure might be a short-lived, very transient state. Second, the middle loop structure was different and did not overlap with those of other arrestins (Fig. 4a, middle panel). Third, the lariat loop moved most closely to the $\mathrm{N}$-domain and made van der Waals contacts with C7pp1 (Fig. 4a, right panel). Lys296 (the corresponding residue of Lys294 in Barr1) belonging to the lariat loop moved toward C7pp1, which might have provided an additional driving force for lariat loop arrangement (Fig. 4a, right panel). Fourth, the C-loop, which was crucial in interacting with GPCR core, resided in similar positions of inactive $\beta$ arr1 and $\beta$ arr2, but not the same position (Fig. 4a, left panel). In addition, due to the different inter- 
domain rotation (Fig. 3), the relative positions of the C-domain were significantly different from each other. We also observed the disruption of the $3 \mathrm{E}$ and the polar-core interactions (Fig. 4b-c). Despite the intrinsic flexibility of each loop containing the central crest, the conformations of the six $\beta$ arr2-C7pp1 molecules in the asymmetric unit matched exceedingly well with each other (Supplementary Fig. 1b), suggesting that each conformation was not derived from a crystallographic artifact, but were the consequences of $\beta$ arr2 activation by C7pp1 binding. Given that these loops were distributed across the surface of $\beta$ arr2, different phosphorylation patterns of the GPCR $\mathrm{R}_{\mathrm{p}}$-tail might induce distinct conformations of $\beta$ arr2 in a combinatorial manner. Collectively, our structure does not overlap with previously determined structures of $\beta$ arr1 or ßarr2, reflecting the high flexibility of arrestins.

Distinct binding modes of C7pp1 compared to other $\mathbf{R}_{\mathbf{p}}$-tails. When we examined the conformations of six C7pp1 peptides in a crystallographic asymmetric unit, two kinds of conformations (chain $\mathrm{U}$ vs chains $\mathrm{V} / \mathrm{W} / \mathrm{X} / \mathrm{Y} / \mathrm{Z}$ ) were observed with slightly different modes of ßarr2 recognition (Supplementary Fig. 1c). The observation suggested that there could be an ensemble of multiple conformations of $\mathrm{C} 7 \mathrm{pp} 1$ when it interacts with positively charged residues distributed on the surface of $\beta$ arr2 (Supplementary Fig. 2). Given that the N-domain of $\beta$ arr2 should interact with hundreds of different patterns of the GPCR $\mathrm{R}_{\mathrm{p}}$-tail, the complex between them might be modular, which has often been observed in disordered proteins ${ }^{25,26}$. The large dependence of electrostatic interactions between $\beta$ arr2 and $R_{p}$-tails might enable $\beta$ arr2 to pair promiscuously with hundreds of GPCRs containing differently phosphorylated $\mathrm{R}_{\mathrm{p}}$-tails.

To investigate how the binding mode of C7pp1 is distinct from those of $\mathrm{V}_{2} \mathrm{Rpp}$ and the rhodopsin C-tail, we compared the conformation of these different structures (Fig. 5a-b and 
Supplementary Fig. 2c-d). For the structural comparisons, we chose the C7pp1 (chain U) bound to $\beta$ arr2 with chain A. It was reported that the phosphopeptides overlapped reasonably well when the structure of the rhodopsin-arrestin complex was superimposed with that of the $\beta \operatorname{arr} 1-\mathrm{V}_{2} \mathrm{Rpp}$ complex $^{10}$. However, when we superimposed the $\beta$ arr2-C7pp1 complex with the $\beta$ arr $1-V_{2} R p p$ complex, the overall conformations of $\mathrm{C} 7 \mathrm{pp} 1$ and $\mathrm{V}_{2} \mathrm{Rpp}$ were significantly different (Fig. 5a). The N-terminal part of C7pp1 was closer to the $\beta 7 / \beta 8$ loop compared to that of $\mathrm{V}_{2} \mathrm{Rpp}$, whereas the C-terminal part of C7pp1 was shorter (Fig. 5a). Especially in the case of $\beta$ arr1, the Nterminal and $\mathrm{C}$-terminal parts of $\mathrm{V}_{2} \mathrm{Rpp}$ made a continuous $\beta$-sheet with $\beta 4$ and $\beta 1$, respectively, of $\beta$ arr1 by anti-parallel stacking. However, those of C7pp1 did not interact directly with either $\beta 4$ or $\beta 1$ of $\beta$ arr2 (Fig. 5a).

Interaction of phosphopeptide with Barr2. Detailed examination of the phosphate-binding sites gave us further insight into the different binding mode of C7pp1 compared to other $\mathrm{R}_{\mathrm{p}}$ tails. C7pp1 contains three phosphates, which consist of the very frequently observed phosphorylation barcode (PxxPxxP) in the GPCR C-terminus. It has been suggested that three positively charged pockets (pocket $\mathrm{A}$, pocket $\mathrm{B}$, and pocket $\mathrm{C}$ ) recognize the phosphorylated serine or threonine consisting of the PxxPxxP barcode ${ }^{10}$. In fact, pSer357 and pThr360 (the first and second phosphates) of $\mathrm{V}_{2} \mathrm{Rpp}$ are nearly superimposable with pThr336 and pSer338 of the rhodopsin Cterminal tail, which bind to pocket A and pocket B, respectively ${ }^{10}$ (Supplementary Fig. 3a).

The three phosphates of C7pp1 make extensive contact with positively charged residues on $\beta$ arr2 (Fig. 5c). The first, second, and third phosphates (pSer335, pThr338, and pThr341) form a salt bridge with $\beta \operatorname{arr} 2 \operatorname{Arg} 148$ (2.4 ̊) (box P), $\operatorname{Arg} 166$ (2.8 and $3.4 \AA$ ) (box A), and Arg26 (2.5 Å) (box B), respectively. Side chains of many other residues (Lys333, Lys337, 
Gly339, Lys342, Leu343, and Asp345) except the phosphorylation sites (pSer335, pThr338, pThr341) point in opposite directions from the interface of $\beta$ arr2 and C7pp1 (Fig. 5c), suggesting that the side chains of other residues do not contribute largely to $\beta$ arr2 binding.

Interestingly, instead of utilizing the same pockets (A, B, and C) in rhodopsin, the new pocket around Arg148 recognizes the first phosphate (pSer335) (Fig. 5c, box P), while pocket A and pocket B interact with the second and third phosphates (pThr338 and pThr341, respectively) (Fig. 5c, boxes A and B). These results suggest that the binding mode of the PxxPxxP barcode is different in the Barr2-C7pp1 complex. We designated the newly identified pocket, which interacts with the first phosphate (pSer335), as pocket $\mathrm{P}$ (Fig. 5c, box P). Next, we checked whether the three pockets $(\mathrm{P}, \mathrm{A}$, and $\mathrm{B})$ can accommodate the binding of the PxPxxP barcode, for which they are responsible. It seems that the space between the first and second phosphates can accommodate either one or two residues because the nearby Lys161 (Fig. 5c, box P), which is a strictly conserved residue (Fig. 5c), might interact with the first phosphate of the PxPxxP barcode. It should be noted that the phosphate sensor residues (Arg8, Lys10, Lys11, Lys107, and Lys294 in $\beta$ arr1) that make contact with the $V_{2} R p p$ phosphates are not involved in the interactions with $C 7 p p 1^{8,27}$ (Supplementary Fig. 3b and 4). The newly identified phosphate binding pocket, pocket $\mathrm{P}$, may be involved in the different conformational changes of $\mathrm{C} 7 \mathrm{pp} 1$ bound $\beta$ arr2 compared to the $\mathrm{V}_{2} \mathrm{Rpp}$-bound $\beta$ arr1, which needs further investigation. Together, these data suggest that various GPCR $\mathrm{R}_{\mathrm{p}}$-tails with different phosphorylation patterns might bind to arrestins differently, which may in turn provide not only the strengths of the interaction but also the ensuing functional outcomes. 
Concluding remarks. As mentioned earlier, an interesting feature of the atypical chemokine receptors (ACKRs) including CXCR7 is their inability to functionally couple G-proteins while maintaining robust interaction with $\beta$ arrs. Thus, it is tempting to speculate that the conformational differences observed here for $\beta$ arr2 in complex with $\mathrm{C} 7 \mathrm{pp} 1$ when compared to $\mathrm{V}_{2} \mathrm{Rpp}$-bound $\beta$ arr1 may reflect a general feature of ACKRs. However, this possibility remains to be experimentally validated in the future for other ACKRs. It is also important to mention that there exists a significant functional divergence between the two isoforms of $\beta$-arrestins, $\beta$ arr 1 and $\beta$ arr2, as mentioned earlier. Thus, it is also plausible that the conformational differences between $\mathrm{V}_{2} \mathrm{Rpp}$-bound $\beta$ arr1 and $\mathrm{C} 7 \mathrm{pp} 1$-bound $\beta$ arr2 represent the mechanistic basis of this functional divergence. For example, $\beta$ arrs have a direct contribution in agonist-induced ERK activation for $\mathrm{V}_{2} \mathrm{R}$, but for $\mathrm{CXCR} 7$, ERK1/2 activation was not observed ${ }^{18}$. Thus, the C7pp1bound $\beta$ arr2 structure may represent an alternative conformation that is not competent to activate ERK1/2 but does support receptor endocytosis and thus ligand scavenging, as reported earlier. However, this hypothesis would require additional experimentation in the future, including structure determination with C7pp2 and perhaps with a phosphorylated CXCR7.

In conclusion, we present a C7pp1-bound structure of ßarr2 that exhibits key structural differences with the previously determined $\mathrm{V}_{2} \mathrm{Rpp}$-bound $\beta$ arr 1 . These findings shed light on the functional divergence of the two $\beta$ arr isoforms and also underline the conformational flexibility in $\beta$ arrs, which allows them to interact with multiple receptors and mediate distinct functional outcomes. Thus, our data may pave the way for developing a better understanding of receptor$\beta$ arr interaction and signaling. 


\section{Methods}

Crystallization and data collection. Before crystallization, $\beta$ arr $2_{1-356}\left(12 \mathrm{mg} \mathrm{mL}^{-1}\right)$ in buffer B containing $200 \mathrm{mM} \mathrm{NaCl}$ and C7pp1 peptide $\left(70 \mathrm{mg} \mathrm{mL}^{-1}\right)$ in $150 \mathrm{mM}$ Tris $\mathrm{pH} 8.0$ were mixed in a 7:1 volume ratio and incubated at $4{ }^{\circ} \mathrm{C}$ for $1 \mathrm{~h}$. Crystals of the $\beta$ arr $2_{1-356}-\mathrm{C} 7 \mathrm{pp} 1$ complex were grown at $22{ }^{\circ} \mathrm{C}$ using sitting-drop vapor diffusion by mixing $1 \mu \mathrm{L}$ of the protein complex solution with $1 \mu \mathrm{L}$ of $20 \%$ (w/v) PEG 3350, 0.2 M ammonium acetate, and 0.1 M Bis-tris pH 5.5. Crystals were cryoprotected by soaking in Paratone ${ }^{\circledR} \mathrm{N}$ oil (Sigma-Aldrich) and flash frozen in liquid nitrogen. X-ray diffraction data were collected at $100 \mathrm{~K}$ in $1^{\circ}$ oscillations at the BL26B1 beamline of the SPring-8 (Japan). Raw data were processed and scaled using the XDS program suite $^{28}$. Table S1 summarizes the statistics of data collection. The $\beta$ arr $2{ }_{1-356}-\mathrm{C} 7 \mathrm{pp} 1$ complex crystal belonged to the space group $C 2_{1}$, with unit cell parameters of $a=91 \AA, b=127 \AA$, and $c$ $=206 \AA$ (Table S1).

Structure determination and refinement. The structure of the $\beta$ arr $2{ }_{1-356-C 7 p p} 1$ complex was solved by the molecular replacement method using a model of mouse S-arrestin (PDB code 5W0P). A cross-rotational search followed by a translational search was performed using the Phaser program ${ }^{29}$. Subsequent manual model building was carried out using the COOT $\operatorname{program}^{30}$, and restrained refinement was performed using the REFMAC5 program $^{31}$. Several rounds of model building, simulated annealing, positional refinement, and individual $B$-factor refinement were performed. Table S1 lists the refinement statistics. The asymmetric unit of the

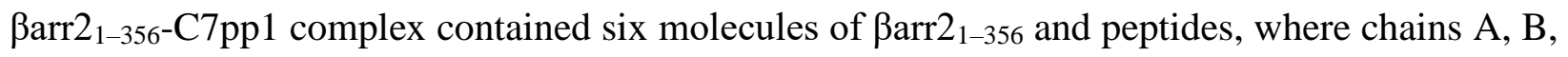
$\mathrm{C}, \mathrm{D}, \mathrm{E}$, and F corresponded to $\beta$ arr2 ${ }_{1-356}$, and chains $\mathrm{U}, \mathrm{V}, \mathrm{W}, \mathrm{X}, \mathrm{Y}$, and $\mathrm{Z}$ corresponded to the 
C7pp1 peptide. This model included 743 water molecules, and $80.4 \%$ of the residues were in the most allowed region of the Ramachandran plot. No electron density was observed for residues 175-181 in chain $\mathrm{C}$ and chain $\mathrm{F}$, respectively.

Accession codes. Crystallographic coordinates of the $\beta$ arr2-C7pp1 complex have been deposited in the RCSB Protein Data Bank with accession number 6K3F. 


\section{References}

1 DeWire, S. M., Ahn, S., Lefkowitz, R. J. \& Shenoy, S. K. Beta-arrestins and cell signaling. Annu. Rev. Physiol. 69, 483-510 (2007).

2 Lefkowitz, R. J., Rajagopal, K. \& Whalen, E. J. New roles for beta-arrestins in cell signaling: not just for seven-transmembrane receptors. Mol. Cell 24, 643-652 (2006).

3 Yang, F. et al. Phospho-selective mechanisms of arrestin conformations and functions revealed by unnatural amino acid incorporation and (19)F-NMR. Nat. Commun. 6, 8202 (2015).

4 Nobles, K. N. et al. Distinct phosphorylation sites on the beta2-adrenergic receptor establish a barcode that encodes differential functions of beta-arrestin. Sci. Signal. 4, ra51 (2011).

5 Xiao, K. et al. Functional specialization of beta-arrestin interactions revealed by proteomic analysis. Proc. Natl. Acad. Sci. U S A 104, 12011-12016 (2007).

6 Reiter, E. \& Lefkowitz, R. J. GRKs and beta-arrestins: roles in receptor silencing, trafficking and signaling. Trends Endocrinol. Metab. 17, 159-165 (2006).

7 Kim, Y. J. et al. Crystal structure of pre-activated arrestin p44. Nature 497, 142-146 (2013).

8 Shukla, A. K. et al. Structure of active beta-arrestin1 bound to a G-protein-coupled receptor phosphopeptide. Nature 497, 137-141 (2013).

9 Kang, Y. et al. Crystal structure of rhodopsin bound to arrestin by femtosecond X-ray laser. Nature 523, 561-567 (2015). 
10 Zhou, X. E. et al. Identification of phosphorylation codes for arrestin recruitment by G protein-coupled receptors. Cell 170, 457-469 (2017).

11 Palczewski, K., Buczylko, J., Imami, N. R., McDowell, J. H. \& Hargrave, P. A. Role of the carboxyl-terminal region of arrestin in binding to phosphorylated rhodopsin. J. Biol. Chem. 266, 15334-15339 (1991).

12 Xiao, K., Shenoy, S. K., Nobles, K. \& Lefkowitz, R. J. Activation-dependent conformational changes in beta-arrestin2. J. Biol. Chem. 279, 55744-55753 (2004).

13 Shukla, A. K. et al. Visualization of arrestin recruitment by a G protein-coupled receptor. Nature 512, 218-222 (2014).

14 Srivastava, A., Gupta, B., Gupta, C. \& Shukla, A. K. Emerging functional divergence of beta-arrestin isoforms in GPCR function. Trends Endocrinol. Metab. 26, 628-642 (2015).

15 Oakley, R. H., Laporte, S. A., Holt, J. A., Caron, M. G. \& Barak, L. S. Differential affinities of visual arrestin, beta-arrestin1, and beta-arrestin2 for G protein-coupled receptors delineate two major classes of receptors. J. Biol. Chem. 275, 17201-17210 (2000).

16 Wei, $H$. et al. Independent beta-arrestin2 and $G$ protein-mediated pathways for angiotensin II activation of extracellular signal-regulated kinases 1 and 2. Proc. Natl. Acad. Sci. U S A 100, 10782-10787 (2003).

17 Zimmerman, B. et al. Role of $\beta$-arrestins in bradykinin B2 receptor-mediated signalling. Cell. Signal. 23, 648-659 (2011).

18 Rajagopal, S. et al. Beta-arrestin- but not G protein-mediated signaling by the "decoy" receptor CXCR7. Proc. Natl. Acad. Sci. U S A 107, 628-632 (2010). 
19 Kroeze, W. K. et al. PRESTO-Tango as an open-source resource for interrogation of the druggable human GPCRome. Nat. Struct. Mol. Biol. 22, 362-369 (2015).

20 Hoffmann, F. et al. Rapid uptake and degradation of CXCL12 depend on CXCR7 carboxyl-terminal serine/threonine residues. J. Biol. Chem. 287, 28362-28377 (2012).

21 Cahill, T. J., 3rd et al. Distinct conformations of GPCR- $\beta$-arrestin complexes mediate desensitization, signaling, and endocytosis. Proc. Natl. Acad. Sci. U S A 114, 2562-2567 (2017).

22 Ghosh, E. et al. Conformational sensors and domain-swapping reveal structural and functional differences between $\beta$-arrestin isoforms. bioRxiv (2019).

23 Kumari, P. et al. Functional competence of a partially engaged GPCR- $\beta$-arrestin complex. Nat. Commun. 7, 13416 (2016).

24 Scheerer, P. \& Sommer, M. E. Structural mechanism of arrestin activation. Curr. Opin. Struct. Biol. 45, 160-169 (2017).

25 Miskei, M. et al. Fuzziness enables context dependence of protein interactions. FEBS Lett. 591, 2682-2695 (2017).

26 Sente, A. et al. Molecular mechanism of modulating arrestin conformation by GPCR phosphorylation. Nat. Struct. Mol. Biol. 25, 538-545 (2018).

27 Gurevich, V. V. \& Gurevich, E. V. The molecular acrobatics of arrestin activation. Trends Pharmacol. Sci. 25, 105-111 (2004).

28 Kabsch, W. XDS. Acta Crystallogr. D Biol. Crystallogr. 66, 125-132 (2010).

29 McCoy, A. J. et al. Phaser crystallographic software. J. Appl. Crystallogr. 40, 658-674 (2007). 
30 Emsley, P. \& Cowtan, K. Coot: model-building tools for molecular graphics. Acta Crystallogr. D Biol. Crystallogr. 60, 2126-2132 (2004).

31 Murshudov, G. N., Vagin, A. A. \& Dodson, E. J. Refinement of macromolecular structures by the maximum-likelihood method. Acta Crystallogr. D Biol. Crystallogr. 53, 240-255 (1997). 


\section{Acknowledgements}

The authors thank the staff at Beamline 7A of the Pohang Light Source and BL26B1 beamline of SPring-8 (Japan) for their assistance during the X-ray experiments. This study was supported by a grant from the National Research Foundation of Korea funded by the Korean government (2015R1A5A1008958 and 2018R1A2B2008142) (H.H.L.), (NRF-2019R1A5A202734011) (K.Y.C.), (2018R1D1A1B07040808) (H.J.Y.), and a grant from the Korea CCS R\&D Center (KCRC; 2014M1A8A1049296) (H.H.L.). The research program in Dr. Shukla's laboratory is supported by an Intermediate Fellowship of the Wellcome Trust/DBT India Alliance Fellowship (grant number IA/I/14/1/501285) awarded to AKS, the Science and Engineering Research Board (SERB) (EMR/2017/003804), the Innovative Young Biotechnologist Award from the Department of Biotechnology (DBT) (BT/08/IYBA/2014-3), and the Indian Institute of Technology, Kanpur. Dr. Shukla is an Intermediate Fellow of Welcome Trust/DBT India Alliance, EMBO Young Investigator, and Joy Gill Chair Professor.

\section{Author contributions}

KM and HJY solved the crystal structure. MB designed and generated CXCR7 phospho-site mutations and performed confocal microscopy. MB and HM carried out the co-IP experiments with CXCR7. HM and JM measured the reactivity of Fab30 with C7pp1/2-bound $\beta$ arr2 using coIP and ELISA. AKS supervised the experiments performed by MB, HM, and JM, and contributed in writing and editing the manuscript. JYP generated the $\beta$ arr2 constructs and performed HDX-MS. KYC supervised the experiments performed by JYP and contributed in writing and editing the manuscript. KM, HJY, KYC, AKS, and HHL analyzed the data and wrote the manuscript. HHL directed the teams and all authors edited the manuscript. 
bioRxiv preprint doi: https://doi.org/10.1101/785527; this version posted September 29, 2019. The copyright holder for this preprint (which was not certified by peer review) is the author/funder. All rights reserved. No reuse allowed without permission.

\section{Conflict of interest}

The authors declare no competing financial interests. 
a

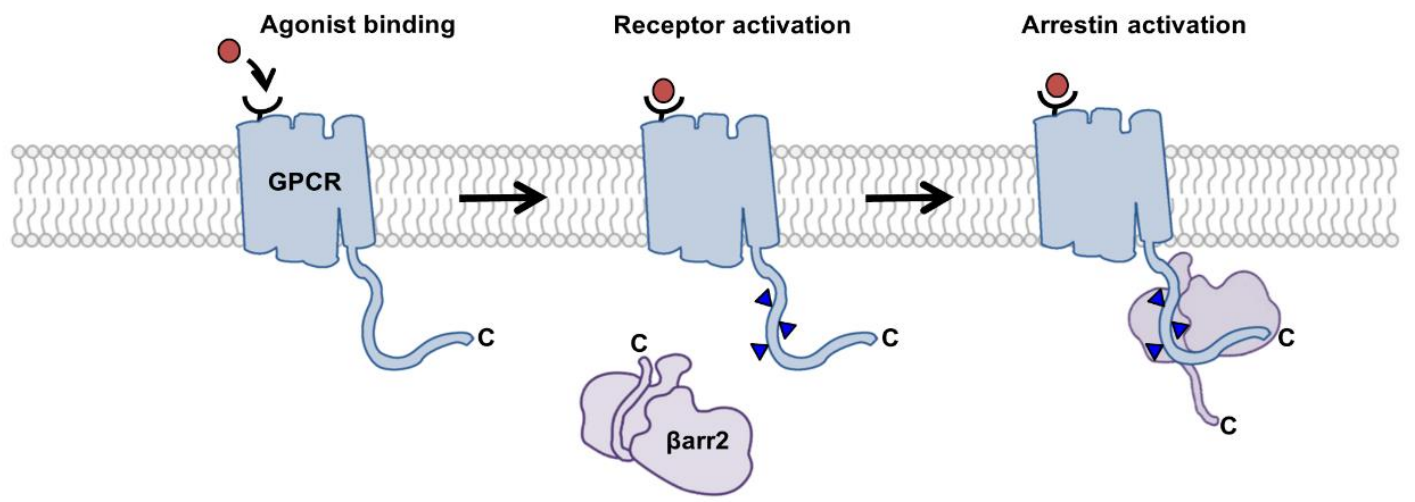

b

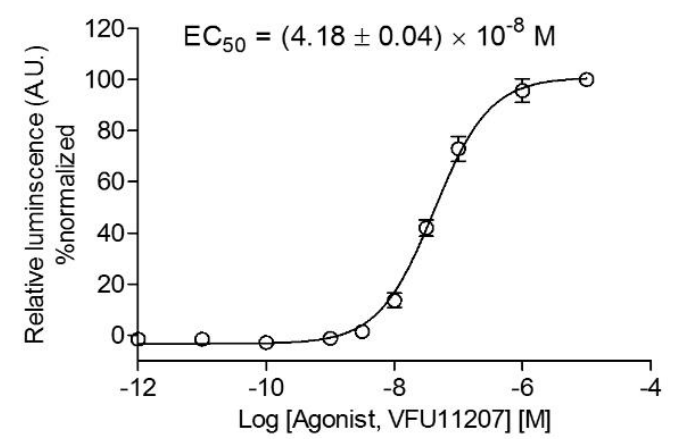

d

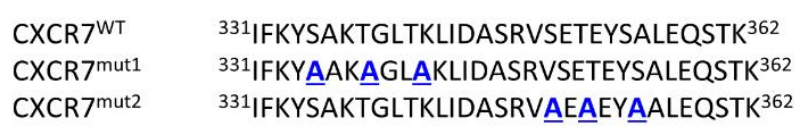

e

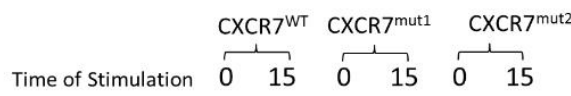

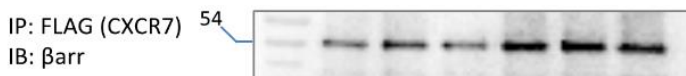
IB: $\beta$ arr IP: FLAG (CXCR7) 54 IB: FLAG (CXCR7)

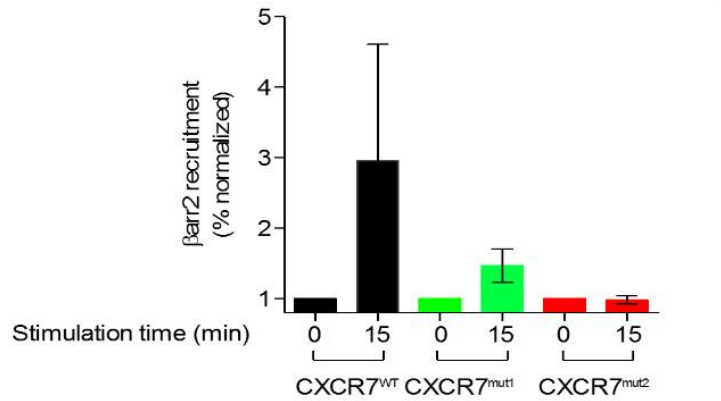

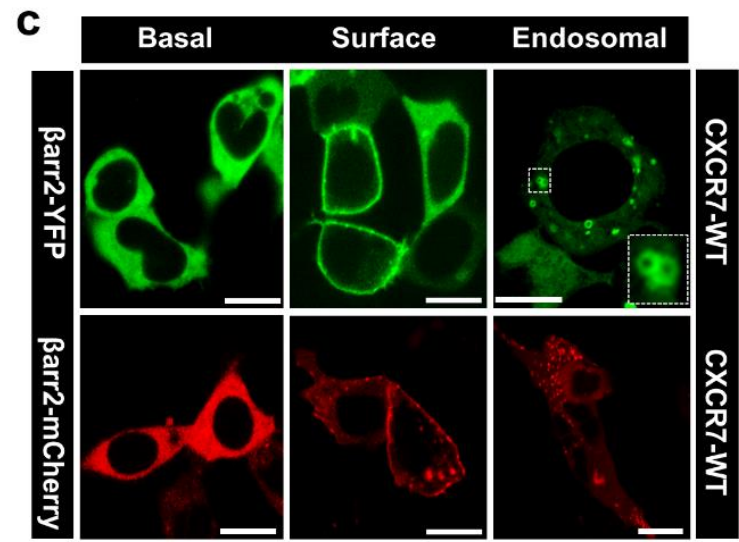

f

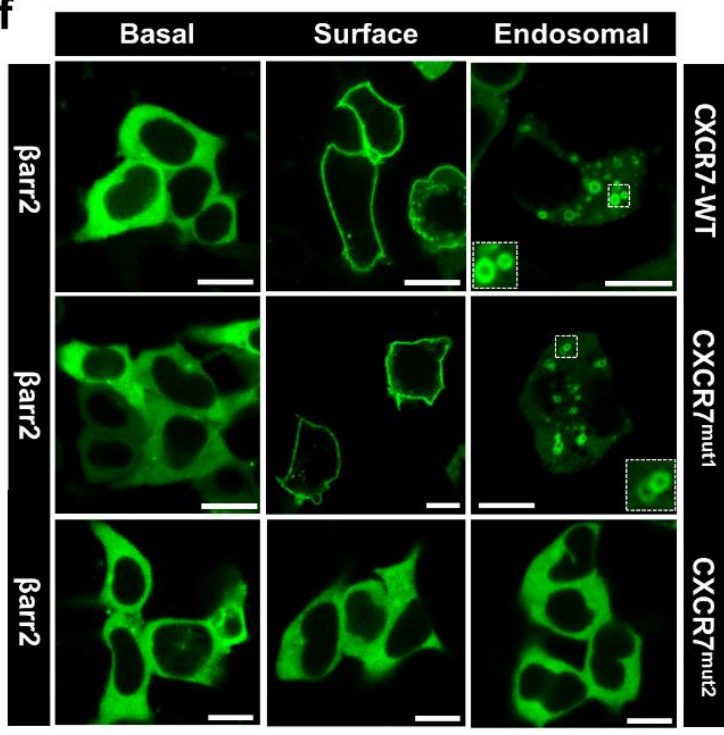

Fig. 1. Agonist-induced Barr2 recruitment and trafficking for the human CXCR7. a, A schematic representation of the typical $\beta$ arr2 recruitment to phosphorylated GPCRs. $\beta$ arr2 interacts with the phosphorylated carboxyl-terminus GPCRs (referred to as receptor tail) first, 
which displaces the carboxyl-terminal tail of $\beta$ arr2 docked in the N-domain. Afterwards, $\beta$ arr2 engages with the intracellular surface of the seven transmembrane bundle (referred to as receptor core) though multiple loops. These two steps of engagement lead to the formation of partiallyengaged and fully-engaged complexes, respectively. b, Agonist-induced recruitment of $\beta$ arr 2 for CXCR7 as measured by PRESTO-TANGO assay. HTLA cells expressing N-terminal FLAGtagged CXCR7 were stimulated with an indicated concentration of agonist (VUF11207), followed by measurement of the luminescence output as a readout of $\beta$ arr 2 recruitment. The data were normalized with respect to maximal response (treated as 100\%). c, Agonist-induced $\beta$ arr2 trafficking monitored by confocal microscopy. HEK-293 cells expressing CXCR7 together with either $\beta$ arr2-YFP or $\beta$ arr2-mCherry were stimulated with a saturating concentration of agonist for the indicated time-points, followed by imaging using the corresponding wavelengths. d, Schematic representation of the carboxyl-terminus residues of wild-type CXCR7 and the two phosphorylation site mutants generated in this study. e, Co-immunoprecipitation experiments reveal the importance of mutant 2 in $\beta$ arr2 recruitment. HEK-293 cells expressing CXCR7 constructs and $\beta$ arr2 were stimulated with a saturating concentration of agonist for the indicated time-points followed by cross-linking using DSP. Subsequently, the receptor was immunoprecipitated using anti-FLAG M1 agarose and the interaction with $\beta$ arr2 was visualized using Western blotting. The bottom panel shows densitometry-based quantification of data presented in panel e normalized with respect to the maximal response for wild-type CXCR7 (treated as $100 \%$ ). f, Confocal microscopy reveals that CXCR7 ${ }^{\text {mut2 }}$ is significantly compromised in inducing $\beta$ arr2 trafficking upon agonist-stimulation while CXCR7 ${ }^{\text {mut1 }}$ behaves mostly like wild-type CXCR7. 
a

\begin{tabular}{|c|c|}
\hline $\begin{array}{l}\text { C7pp1 } \\
\text { PxxPxxP }\end{array}$ & 331 \|FKY \\
\hline $\begin{array}{l}\text { C7pp2 } \\
\text { PxPxxP }\end{array}$ & ${ }^{346}{ }_{\text {ASRVṠET̈EYS̈ALEQ }}^{359}$ \\
\hline
\end{tabular}

- Proximal phosphate

.. Middle phosphate

... Distal phosphate b

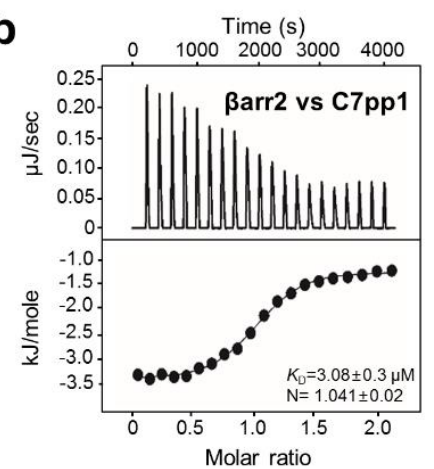

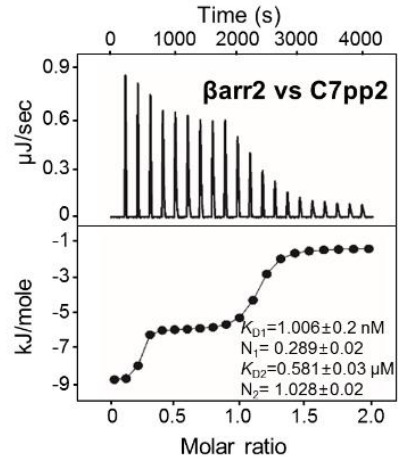

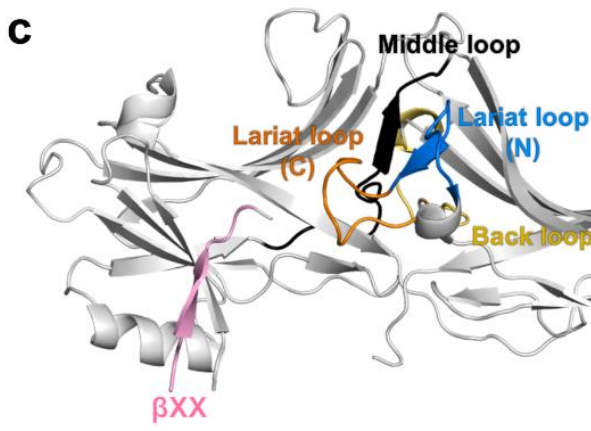

Lariat loop (C)
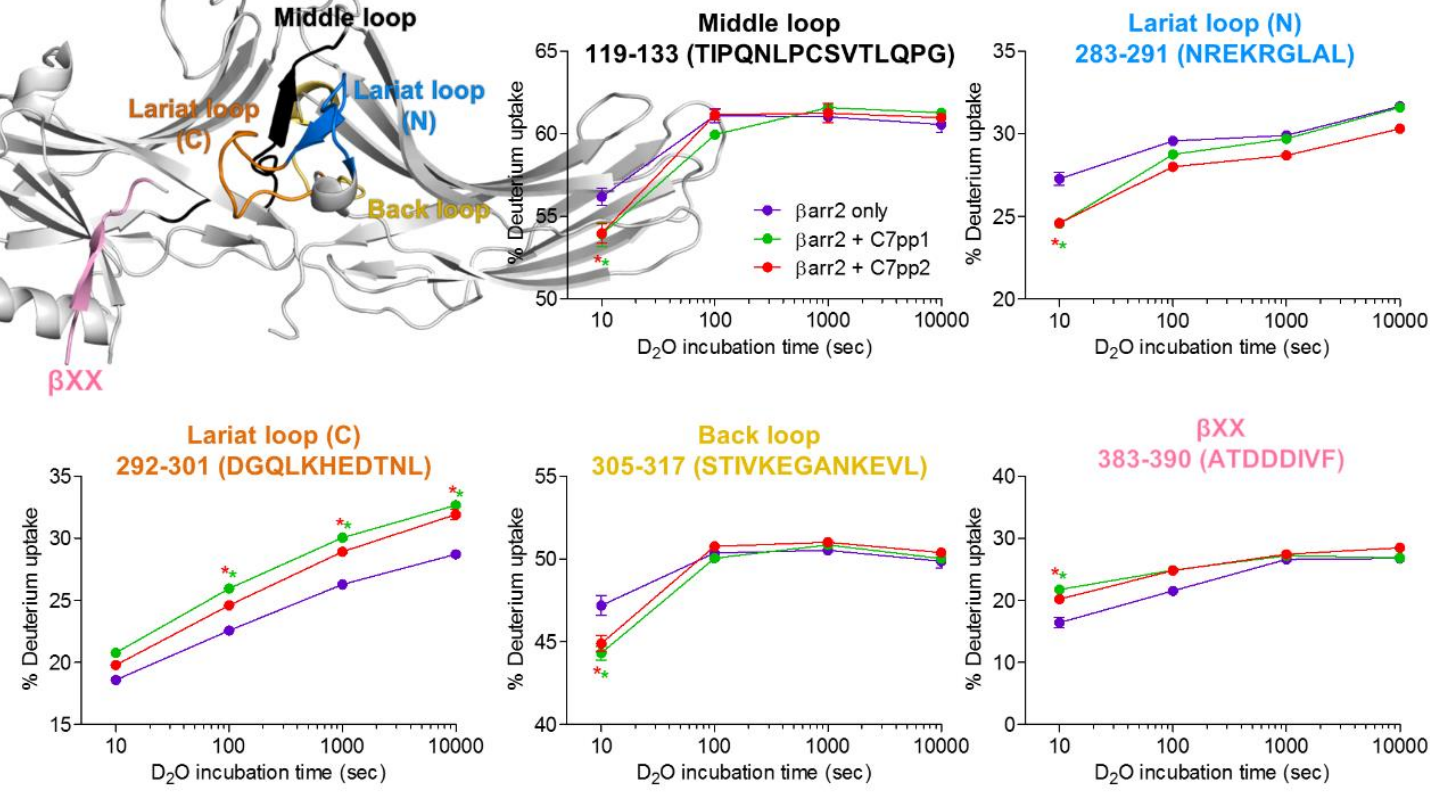

d

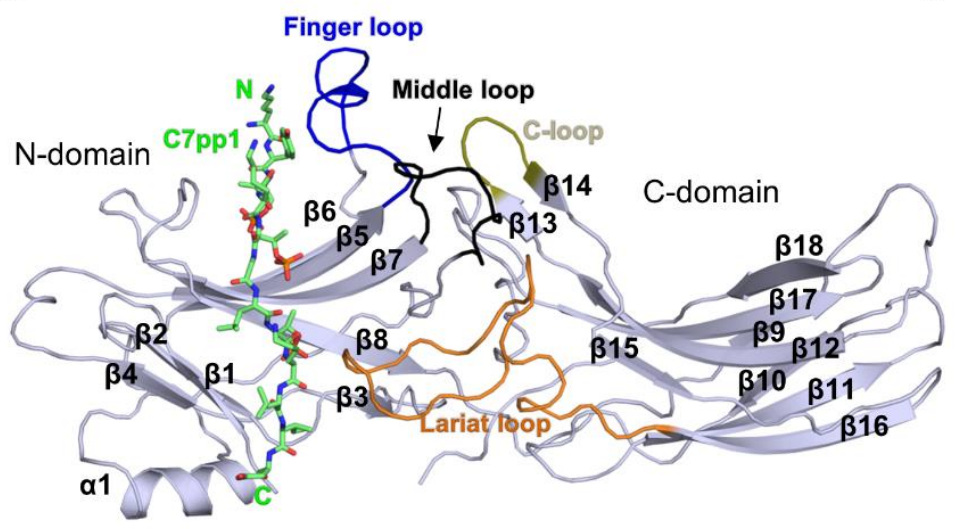

e $\mathrm{k} 333$
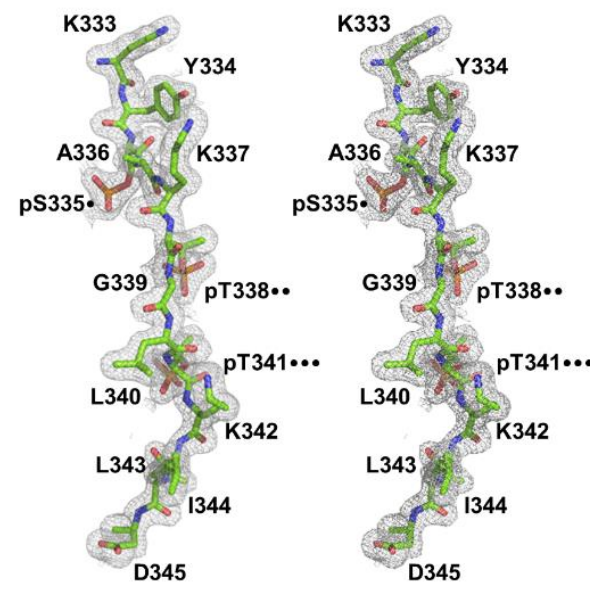

Fig. 2. CXCR7 phosphopeptides and crystal structure of $\beta$ arr2 in complex with C7pp1. a,

The peptide sequences of the CXCR7 phosphopeptides referred to as C7pp1 and C7pp2 
(hereafter, colored in green and red, respectively). The positions of proximal, middle, and distal phosphates of either PxxPxxP or PxPxxP phospho-barcodes are denoted in dots. b, Binding affinity of CXCR7 phosphopeptides with $\beta$ arr2 as measured by isothermal calorimetry. Purified Barr2 was incubated with increasing concentration of the individual phosphopeptides and the binding parameters were calculated based on the dose response curve. The binding constant for each peptide and stoichiometry as observed in three independent experiments is presented here. c, HDX-MS profile of $\beta$ arr2 upon C7pp1 or C7pp2 binding. Regions with altered HDX profile are color-coded on the inactive structure of $\beta$ arr2 (PDB ID: 3P2D), and the deuterium uptake plots of color-coded regions are provided. Data represent the mean \pm standard error of the mean of three independent experiments. The statistical analysis was performed using one-way ANOVA followed by Tukey's post-test (* $p<0.05$ compared to $\beta$ arr2 alone). Differences smaller than 0.3 Da were not considered significant. d, Overall structural snapshot of C7pp1-bound ßarr2 highlighting the loop regions. The C7pp1 peptide is shown as green sticks and the various loops in ßarr2, i.e., the finger, middle, lariat, and C-loops in the central crest, are colored in blue, black, orange, and olive, respectively. e, The stereo $2 \mathrm{Fo}-\mathrm{Fc}$ map for $\mathrm{C} 7 \mathrm{pp} 1$ is drawn with a 1.0 sigma contour. The positions of proximal, middle, and distal phosphates of the phospho-barcode $(\mathrm{PxxPxxP})$ are denoted in dots. 


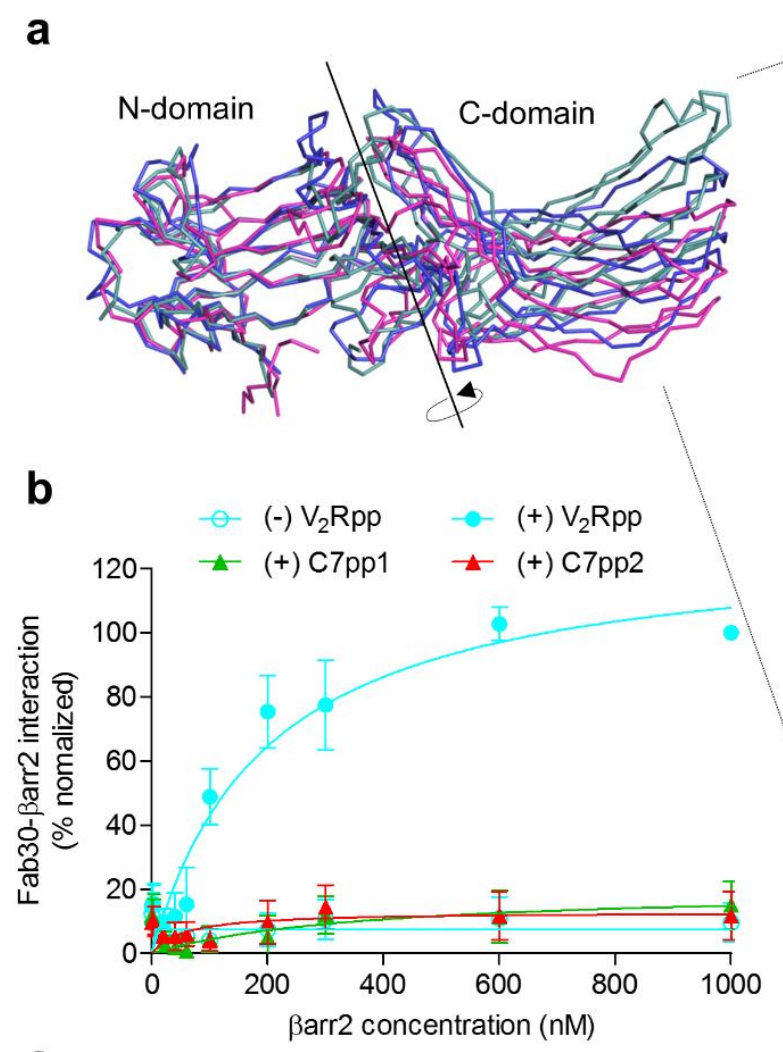

C

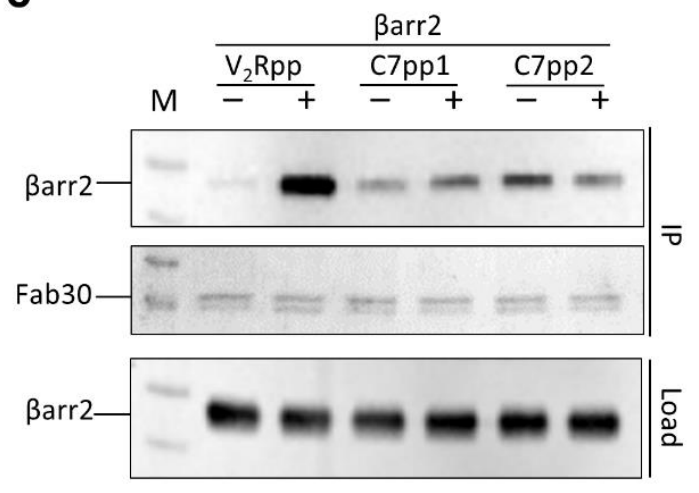

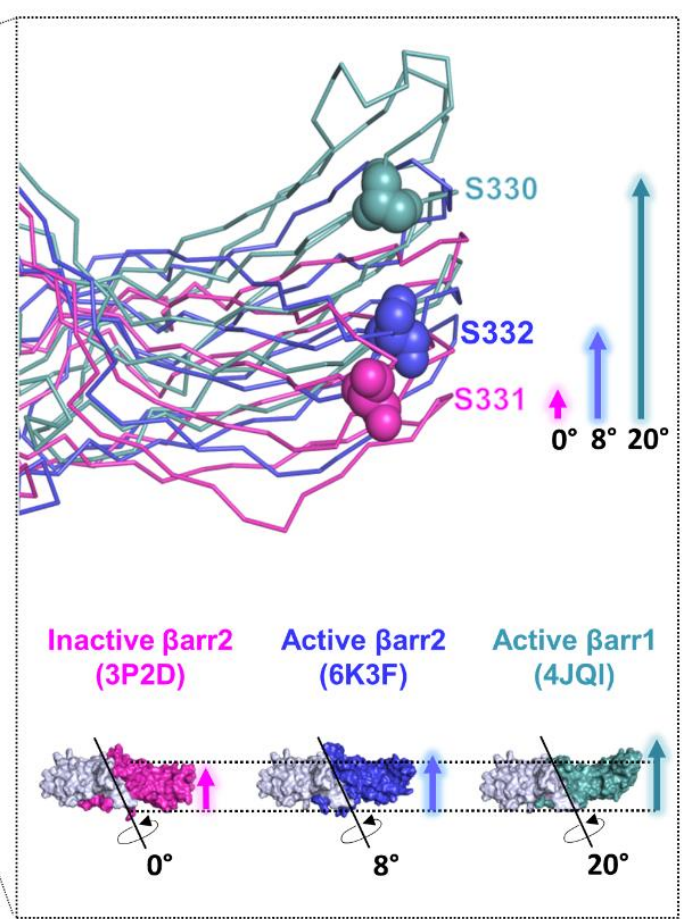

d

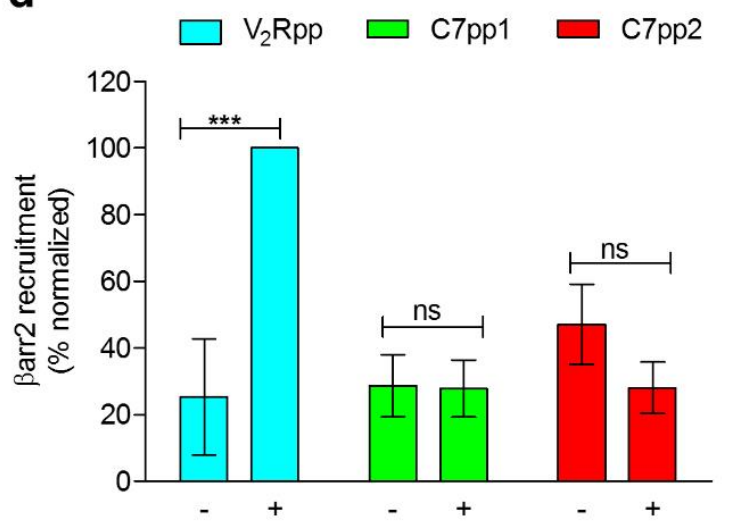

Fig. 3. C7pp1-bound ßarr2 exhibits a smaller inter-domain rotation compared to $V_{2} R p p-$

Barr1 and the Fab30 sensor corroborates the observation. a, Inter-domain rotation comparison of $\mathrm{V}_{2}$ Rpp-bound $\beta$ arr1 (light cyan, PDB ID: 4JQI) and C7pp1-bound $\beta$ arr2 (blue, PDB ID: 6K3F) with the corresponding inactive states of $\beta$ arr2 (magenta, PDB ID: 3P2D). The $\mathrm{N}$-domains are superimposed, and the rotation axis is indicated in the magnified view of the $\mathrm{C}$ domain. The relative positions of $\mathrm{Ser}^{332}$ of active $\beta$ arr2 (PDB ID: 6K3F) are shown in ball 
representation as a reference for comparison. The crystal structure of $\beta$ arr2 in complex with C7pp1 (blue) reveals an inter-domain rotation of about $8^{\circ}$ compared to the inactive $\beta$ arr2 structure (magenta), suggesting an intermediate active state. b, The Fab30 reactivity pattern

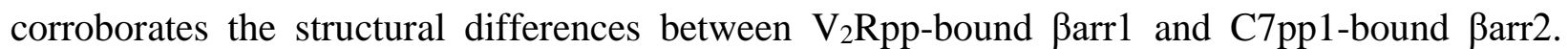
Increasing concentrations of $\beta$ arr2 in the presence of a saturating concentration of different phosphopeptides were immobilized on an ELISA plate followed by incubation with Fab30 and detection using HRP-coupled Protein L. Data were normalized with the maximal response for $\mathrm{V}_{2} \mathrm{Rpp}$ - $\beta$ arr2 condition (treated as $100 \%$ ). c, Co-immunoprecipitation experiments further confirm the Fab30 reactivity patterns as observed in ELISA. Purified $\beta$ arr2 was incubated with a saturating concentration of different phosphopeptides followed by addition of 1.5-fold molar excess of Fab30. Afterwards, Fab30 was immunoprecipitated using Protein-L agarose and the interaction of Fab30 and $\beta$ arr2 was visualized using Western blotting. A representative image from three independent experiments is shown here. $\mathbf{d}$, Densitometry-based quantification of data presented in panel c normalized with respect to the maximal response for the $\mathrm{V}_{2} \mathrm{Rpp}-\beta \mathrm{arr} 2$ condition (treated as $100 \%$ ). Data were analyzed using ONE-WAY ANOVA with Bonferroni post-test $(* * * \mathrm{p}<0.001)$ 


\section{a}

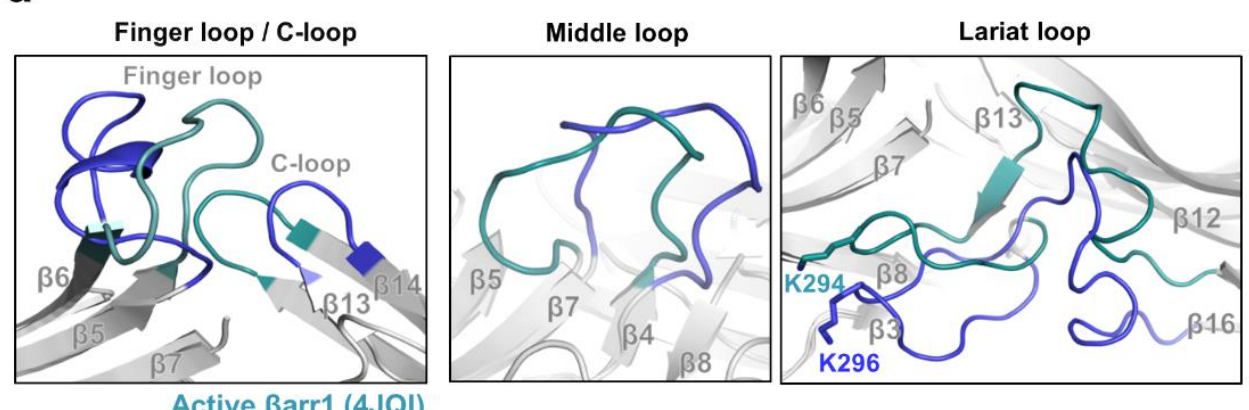

b

Active Barr1 (4JQI)

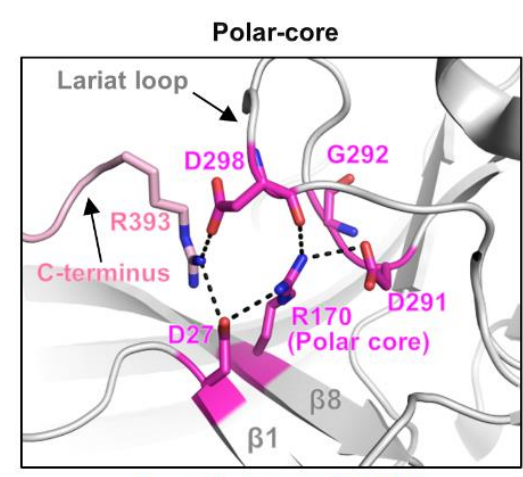

Inactive $\beta$ arr2 (3P2D)

C

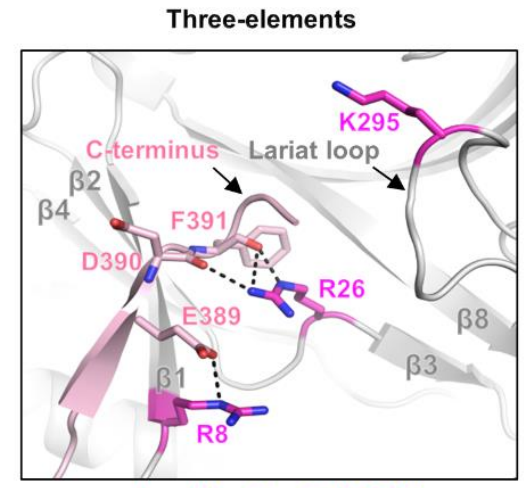

Inactive $\beta$ arr2 (3P2D)

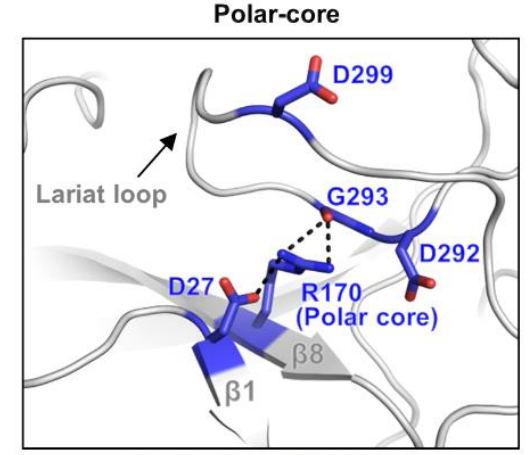

Active $\beta$ arr2 (6K3F)

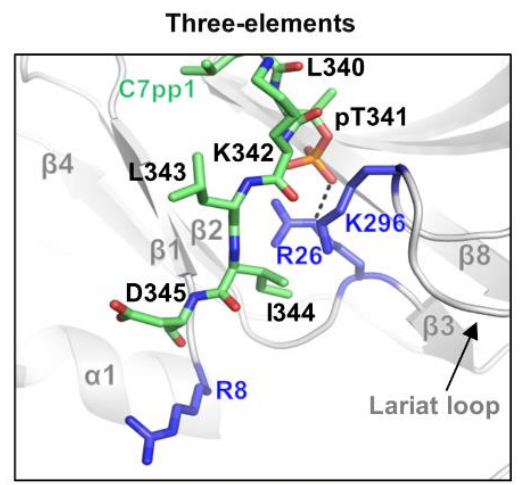

Active $\beta$ arr2 (6K3F)

Fig. 4. Conformational changes in various loops of ßarr2 upon C7pp1 binding as observed

in the crystal structure. a, Structural comparisons of the finger, middle, lariat, and C loops in C7pp1-bound $\beta$ arr2 (6K3F) and $\mathrm{V}_{2} \mathrm{Rpp}$-bound $\beta$ arr1 (PDB 4JQI). b-c, Structural comparisons of polar core and 3E interactions in inactive $\beta$ arr2 (3P2D, magenta) and C7pp1-bound $\beta$ arr 2 (6K3F, blue), respectively. The C-terminus and C7pp1 are colored in pink and green, respectively. 
a

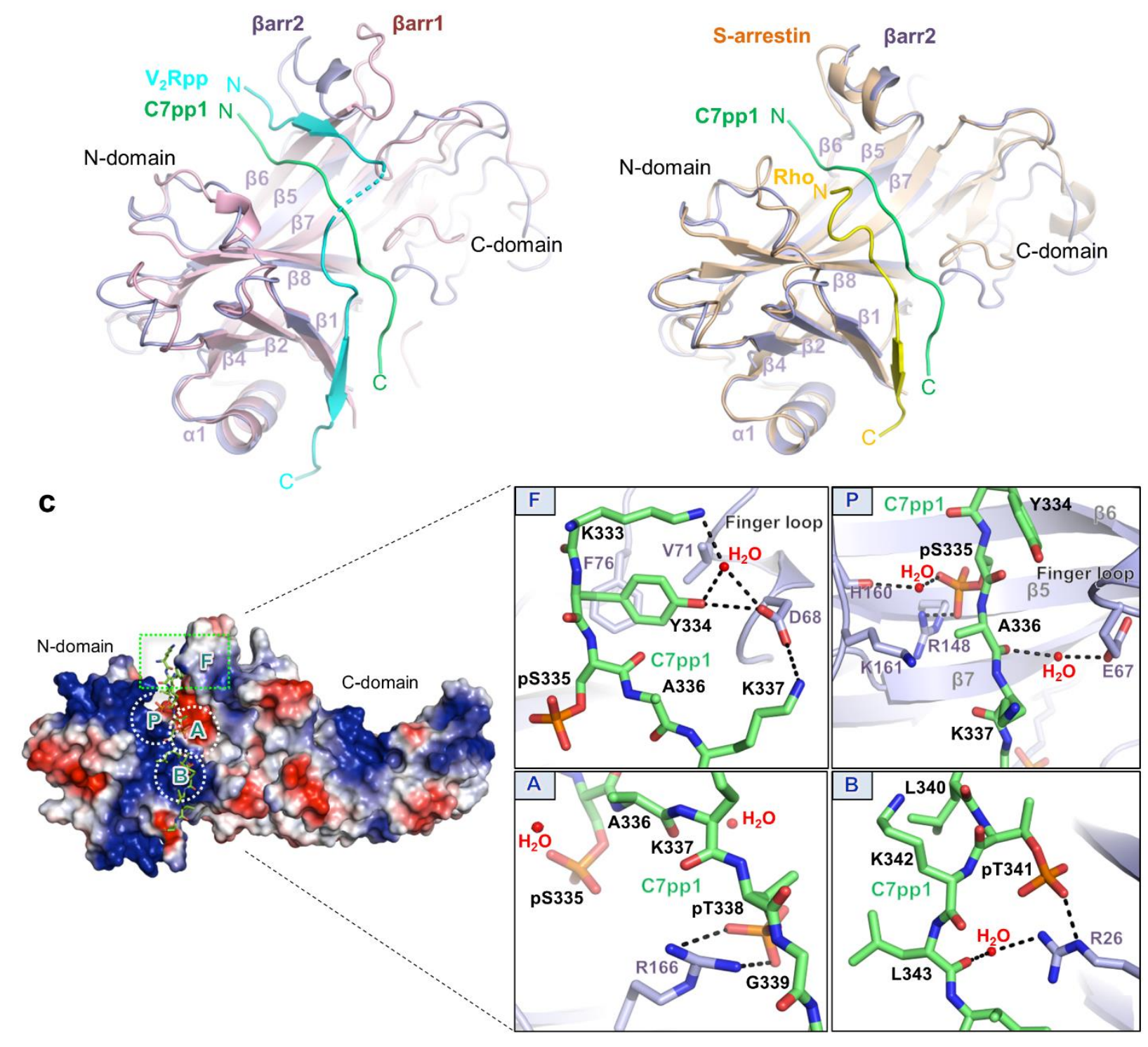

Fig. 5. Overall binding mode of C7pp1 to $\beta$ arr2 with specific interactions of the phosphate groups and activation switches. a, An overall distinct binding mode of C7pp1 with ßarr2 (6K3F, green) compared to the $\mathrm{V}_{2} \mathrm{Rpp}-\beta$ arrl complex (4JQI, light cyan). The N-domains from the crystal structures of the C7pp1- $\beta$ arr2 complex and $V_{2}$ Rpp- $\beta$ arr1 are superimposed and the respective phosphopeptides are highlighted for comparison. b, A comparison of binding modes of C7pp1 with $\beta$ arr2 (6K3F, green) and the rhodopsin $R_{p}$ tail with $S$-arrestin (5W0P, yellow) 
presented in a similar fashion as in panel a. c, Surface representation for overall electrostatic potential of the C7pp1-bound $\beta$ arr2 structure. C7pp1 is shown as green sticks. In the positive electrostatic surface of the $\mathrm{N}$-domain, the four hot-spots for $\mathrm{C} 7 \mathrm{pp} 1$ binding are shown in the dotted rectangle or circles $(\mathrm{F}, \mathrm{P}, \mathrm{A}$, and $\mathrm{B})$. The panels on the right represent the detailed interactions at the $\beta$ arr2-C7pp1 interface and specific interactions of the phosphates with various residues in $\beta$ arr2. 